\title{
THE PROBLEM OF MOTION IN MICROWORLD (A TRANSFORMATIONAL ANALYSIS)
}

\author{
"What I am going to tell you about is what we teach our physics \\ students in the third or fourth year of graduate school... It is my task to \\ convince you not to turn away because you don't understand it. You see my \\ physics students don't understand it.... That is because I don't understand it. \\ Nobody does." \\ Richard Feynman
}

We analyze the behavior of particles in the micro world by using theories of quantum mechanics. In quantum mechanics there are numerous philosophical problems connected with micro world, which are not comprehensively defined. However, according to academician G. Brutian's system of transformational logic one can pass from implicit ideas to explicit knowledge by checking corresponding contents of concepts.

Our logical traditions include experiment, which is not applicable for a new phenomena via corresponding scales. There is no chance for future scientific research that this experience could be satisfactory. It is explained that one can't speak about mechanical motion in the sphere of quantum mechanics, since there are no trajectories of electron motion inside the atom.

Academician Georg Brutian his system of transformational logic presented in more than a dozen books and papers, from which I basically have used the monograph "Transformational logic» (Brutian 1983). In his main works G. Brutian shows how many transformational rules we can formulate in the field of formal logic. One of academician Brutian's first followers in this field is R.Djidjian. He has demonstrated in his papers that transformational logic, especially the transformational analysis, has a key role in the field of the theory of argumentation (Djidjian 1988). Further, R.Djidjian has also showen that transfor- mational logic can be considered as a method of scientific research as well as a guideline in creating artificial intellect (Djidjian 2008). The works of new generation of armenian philosophers extended G.Brutian's theory of transformational logic. Their results were published in two volumes of "Transformational logic" (Brutian 2008). Here are of special interest two papers of Anna Amirkhanyan devoted to the analysis of different aspects of formal logic and mathematical logic in the light of transformational logic. One can be sure that the research of young scientists in this field should open new ways for expension of academic Broutian's system of transformational logic.

Transformational logic can be very helpful for correcting the meaning and use of the concepts of space, time, and motion. The need of such research is especially acute when researching the phenomena of the micro world since one can't use here such basic concepts as the inner special structure of micro objects and the concept of trajectory of elementary particles that compose them. In this regard it should be useful to suggest a new interpretation for the principles of complementarity and correspondence in the light of the most recent discoveries in the physics of elementary particles as well as in the light of the transformational logic. 
1. Motion in the microworld and Nils Bohr postulates

The planetary model of atom was suggested by Ernst Rutherford in 1908. Rutheford classical atom is unstable. According to the laws of classical physics, electrons, which move with aceleration by corresponding orbit, after short period $\left(10^{-8} \mathrm{sec}\right)$ should fall dawn on the nucleus: radiate light with corresponding wave length. However it is not correct.

The next step is about the theory of atom's structure, realized by Nils Bohr. By investigating the contradiction between Rutherford's contradictory model of atom and the laws of classical physics, and by analysing all experimental data, Nils Bohr suggested in 1913 his famous "postulates", which explained the structure and properties of hydrogen atom. Deliberating over his theory, N.Bohr came to the conclusion, that for correctly describing the behaviour of atomic systems one should reject a number of concepts of classical physics. (Bohr 1970)

The postulates of N.Bohr show that atoms "live" according the laws of the microworld, which are principally different from our common sense understanding of physical reality. Bohr's first postulate proves the existence of stationary states. In an atom, the electrons revolve around the nucleus in certain definite circular paths called orbits, or shells. The second postulate proves that each electron orbit corresponds to a definite energy. The circular orbits of atomic electrons are also known as energy levels.

The electrons in an atom can move from a lower energy level $E_{1}$ to a level of higher energy $E_{2}$ by absorbing the appropriate amount (or quantum) of energy. Similarly, an electron can jump from a higher energy level $E_{2}$ to a lower energy level $E_{1}$ by losing the appropriate quantity of energy. The energy absorbed or lost is equal to the difference between the energies of the two energy levels, i.e., $\Delta E=E_{2}-E_{1}$.
The third postulate proves that the frequency of atomic radiation (absorption) of energy is defined by the energetically difference of stationary states $h \cdot v=E_{m}-E_{n}$ (here $\left(E_{m}-E_{n}\right)$ is the difference of energies of stationary states, $k$ and $\mathrm{n}$ - are the main quantum numbers, which describe the stationary states.

Actually, by verifying experimentally Rutherford's model of atom, taking into account postulates of Bohr, scientists reveal the limits of the laws of classical physics for the bodies of micro world.

In the light of Academician Brutian's transformational logic, the progress from classical electrodynamics to quantum electrodynamics can be presented as a procedure of explication of inner implicit contradictions of classical theory and transformation of classical continuous concepts into discrete concepts of quantum mechanics.

Another important implicit point of the microworld is that In the atom, motion of electrons aren 't like orbital motion of planets or other macro bodies. We can not determine the exact position of an electron on the orbit: we only can determine the probability of finding an electron just a given point (or rather section) of the orbit (Bohr 1971).

2. The concept of orbit and quantum mechanics

The foundations of quantum ideas developed by M.Plank presume that energy of electromagnetic radiation get discrete values. Then A.Einstein using the discovery of M.Plank explained the phenomenon of photoelectric effect. Further, Louis de Broglie put forward the principle of wave-particle duality. The basic theory of quantum mechanics was developed by Erwin Schrodinger and Werner Heisenberg (Vasilevsky 1991: 17).

The investigation of quantum physics provides solutions for many new problems. And we can show that conditions by which laws of classical physics are applicable depend on the value 
of energy involved in phenomena under investigation. Namely classical physics is applicable to phenomena and processes where values of interaction are much bigger compared with the $M$. Plank quantum of action ( $h=6,62 \cdot 10^{-27} \mathrm{erg} \cdot \mathrm{sec}$ ). The contemporary understanding of quantum mechanics allows to solve physical problems, which we couldn 't be solved in classical physics.

Note, that all this we can apply not only in the field of quantum mechanics, but also in the different spheres of science, which is the philosophical meaning of this principle.

Implicit became explicit, but not always, it is defined. For instance: many physical quantities are not exactly defined till now. For instance, motion in the microworld. Therefore, there are no motion and trajectory concepts in the quantum mechanics.

\section{Motion in the nucleon of atom}

Since the positive electrical charge of protons create repulsive forces in the nucleus, there are in the nucleus also neutrons for its stability. In the case of light nuclei their quantity is approximately equal, but in the case of heavy nuclei the quantity of neutrons gradually increases.

Nuclear forces acting between neutrons and protons, compared to electrical forces are stronger, but at the extremely short distances about $10^{-13} \mathrm{~cm}$ that is 100000 time smaller of the size of atoms. The density of matter in the hydrogen nucleus is $10^{14} \mathrm{~g} / \mathrm{cm}^{3}$ (compare: the density of iron is equal to $\left.7 \cdot 10^{3} \mathrm{~g} / \mathrm{cm}^{3}\right)$. By these conditions the idea of mechanical motion of protons and neutrons inside the atomic nuclei loses its sense.

4. The main problem of inner structure of elementary particles and motion

According to the contemporary conceptions, protons and neutrons, and all other elementary particles are built of quark. The interaction of elementary particles, especially in the case of high energy, produces very unexpected results: elementary particles, which have small mass of rest by the result of interaction can create particles, which have much bigger mass of rest. This means that in the field of conversion of elementary particles the conception of the special structure of particles has lost it's meaning. Therefore, also there is no meaning to speak about mechanical motion also "inside" elementary particles.

Conclusion:

Implicit became explicit, but not always it has got strict definition.

Therefore quantum mechanics reach great success in the micro world, in the field atomic and molecular phenomena. Nevertheless the basic problems of quantum mechanics and philosophical generalizations connected with them yet demand to undertake new and fundamental methodological research and conclude it via corresponding definitions. There are a number of philosophical questions, connected with quantum mechanics, which still require clarifications. Usually they are connected with the nature of probability in the micro world.

Quantum physics is the thinking of the new century which bravely confronts contradictions and paradoxes. We are sure the solution of arising problems could be helped by the ideology of George Brutian's system of transformational logic: we must check corresponding contents of concepts and pass from implicit ideas to explicit knowledge.

In the contemporary physics the problem of nucleus has special place, which is one of the interesting branch of physics. In this article we consider some of the casses, we came to the conclusion, that our logical traditions include experiment, which is not applicable for a new phenomena with new scales.

Common sense has accumulated the entire experience of past times. But in regard of future scientific discoveries there is no chance that this experience could be satisfactory. In the field of the physics of the microworld one should all the time take into an account possibilities of encoun- 
tering new unordinary phenomena and not be afraid of them (Feynman 1976).

The above presented transformational analysis demonstrates that we can't speak about mechanical motion in systems and objects of quantum mechanics. Thus there is no place for the

\section{REFERENCES}

1. Bohr, N. (1970). Collected Scientific Works. P. I. M., "Science" (in Russian).

2. Bohr, N. (1971). Collected Scientific Works. P. II. M., "Science" (in Russian).

3. Brutian, G. (ed.) (2008). Transformational logic. Book 1 and Book 2. Yerevan. «International academy of philosophy» publ.

4. Brutian, G. (1987). Course of logic: Yerevan, ed. Yerevan State University (in Armenian).

5. Brutian, G. (1983). Transformational logic. Yerevan: ed. NA Arm. SSR (in Russian).

6. Djidjian, R. (2008). Transformational Logic as a Basis for Machine Understanding. // Brutian, G. (ed.).

7. Transformational logic. Book 2. Yerevan. «International academy of philosophy» publ., (pp. 555-571) (in Russian). concept of trajectory of motion. Explicating the essence of situation, we should be satisfied by presenting the world of elementary particles by energetical descriptions and explanations.

8. Djidjian, R. (2002). The Secret of Geniality. Yerevan: "Noyan Tapan” publ.

9. Djidjian, R. (1988). Transformational logic and argumentation. Questions of philosophy, Yerevan, 1988, № 6, 7, pp. 228-235.

10. Djidjian, R. (1983). Getting ready for great discoveries. Yerevan, Yervan state univ. publ.

11. Feynman, R., Leighton, R., Sands, M. (1966). The Feynman Lectures on Physics, Moscow, 1976, A. (1968). Problems in the Philosophy of Science. Amsterdam.

12. Vasilevski, A.C. (1991). Course of Theoretical Physics, Moscow, (in Russian).

GEVORGYAN MARIAM

\section{THE PROBLEM OF MOTION IN MICROWORLD. \\ A TRANSFORMATIONAL ANALYSIS}

\section{SUMMARY}

Transformational logic can be very helpful for correcting the meaning and use of the concepts of space, time, and motion. The need of such research is especially acute when researching the phenomena of the microworld since one can't use here such basic concepts as the inner special structure of microobjects and the concept of trajectory of elementary particles that compose them. In this regard it should be useful to suggest a new interpretation for the principles of complementarity and corresponddens in the light of the most recent discoveries in the physics of elementary particles as well as in the light of the transformational logic.

Key concepts: transformational logic, motion in the microworld, quantum electrodynamics, quark, photoelectric effect, wave-particle duality, nuclear forces. 


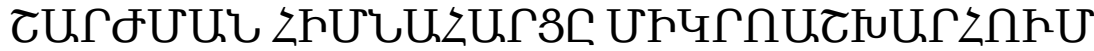

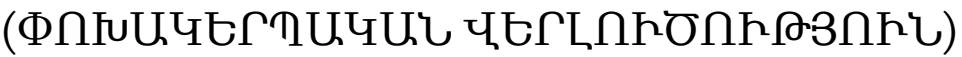

\section{UUФกФПЋU}

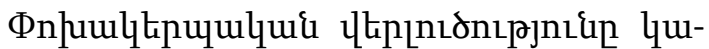

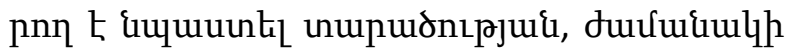

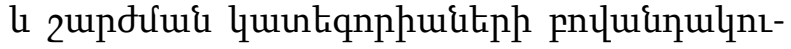

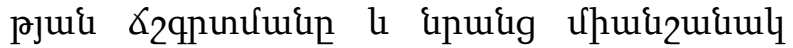

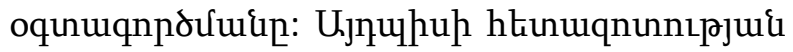

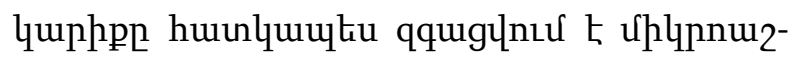

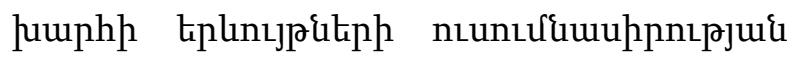

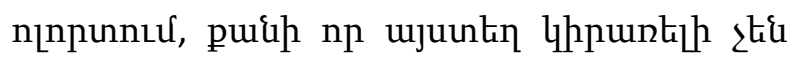

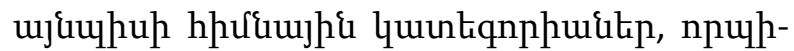

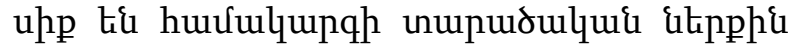

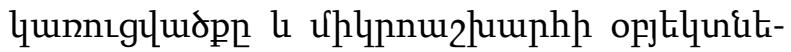

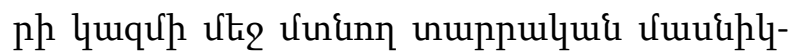

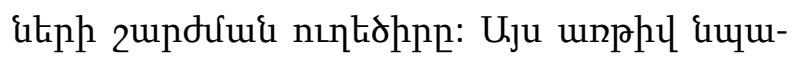

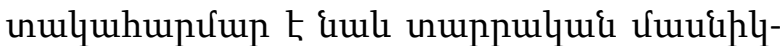

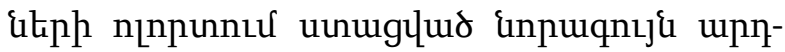

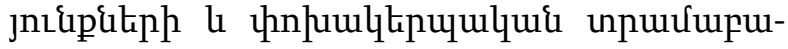

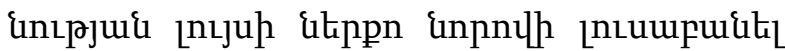

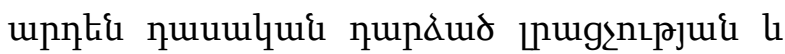

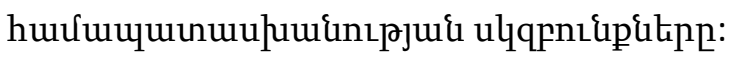

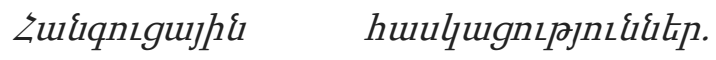

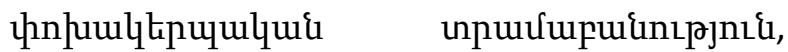

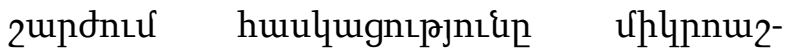

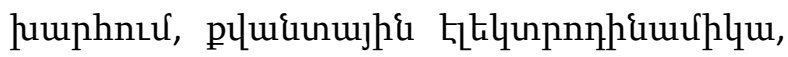

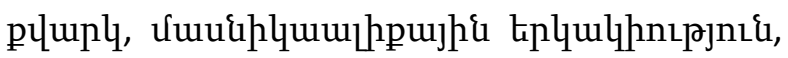
uhenılqujhi nıdin: 